\title{
Novel Communication Services Based on Human Body and Environment Interaction: Applications inside Trains and Applications for Handicapped People
}

\author{
Jordi Agud Ruiz, Shigeru Shimamoto \\ Graduate School of Global Information and Telecommunication Studies, Waseda University \\ 29-7 building, 1-3-10 Nishi-Waseda, Shinjuku-ku, Tokyo 169-0051 Japan \\ E-mail: jordi.agud@toki.waseda.jp,shima@waseda.jp
}

\begin{abstract}
We believe that near-field radio intra-body communications, wherein human body is used as the transmission medium, will be a very suitable solution for Body Area Networks with many interesting applications towards a ubiquitous communication world. This paper presents some potential new applications of great usefulness for handicapped people. Moreover, we have also envisioned applications inside train coaches where the use of mobile phones is restricted in countries such as Japan. On the other hand, we present the system model designed to carry out these applications by using intra-body communications. Finally, in order to investigate the feasibility of this kind of communication, we analyze experimentally the characteristics of the intra-body propagation channels and we evaluate the performance of several digital modulation schemes.
\end{abstract}

Keywords-intra-body communications, body area networks, applications for handicapped people, services inside trains, intrabody channels

\section{INTRODUCTION}

Body Area Networks (BAN) will allow new ways of wireless communications where the user will be able to interact with devices that move with the user or that are part of user's body. One of proposed technologies to achieve the goal of BAN is intra-body communications (IBC), a short-range communication solution between devices in which the human body is used as the signal transmission medium. We believe that IBC will allow not only the interaction between devices that move with the user body but also between users and the surrounding environment. There are two main solutions for IBC: electric field type [1] and [2] and electromagnetic type [3]. By means of the former solution, data is sent across devices placed on or near the body by using a near field electric field. In the latter solution, the human body is treated as a waveguide with the radio frequency signals propagating through the body. Our research is based on the near-field radio solution whereby we aim to provide broadband IBC in order to satisfy future new applications (such as transferring multimedia files through the body) that will require high data rates.

Related works have foreseen applications using IBC such as exchange of business cards between two people by handshake [1], open a car door by touching the knob [2] and personalization of wearable computers [3]. In this paper, we present novel applications (guidance system, location based information and e-payment) for handicapped people (blind and deaf people). Moreover, we introduce applications in which IBC could be employed in places such as train coaches and hospitals where the use of mobile phones is not allowed. We also show how users could get personalized information based on their interests and requirements by means of a simple touch of an IBC enabled information source. The paper is organized as follows. Section II describes the IBC system model. Afterwards, Sections III and IV present the applications for handicapped people and the applications inside train coaches respectively. Section V investigates experimentally the transmission characteristics of the intra-body propagation channels and Section VI evaluates the performance of three digital modulation schemes applied to the intra-body channels. Finally, Section VII contains the conclusions of this paper.

\section{Proposed IBC System ModeL}

We differentiate three different kinds of elements in our IBC system model: central unit (CU), wearable devices and environment sensors. Fig. 1 shows an example scenario where the user wears a watch-type in his wrist (i.e. the CU), eye-glass display, earphones, mobile phone, a sensor for monitoring heart pulse and a foot-sensor device for environment sensing. Moreover, an environment sensor is located in the ground. All the devices in the Fig. 1 are IBC enabled. Furthermore, some devices could also carry out communication outside the body (e.g. mobile phone in Fig. 1), that is, short range and long

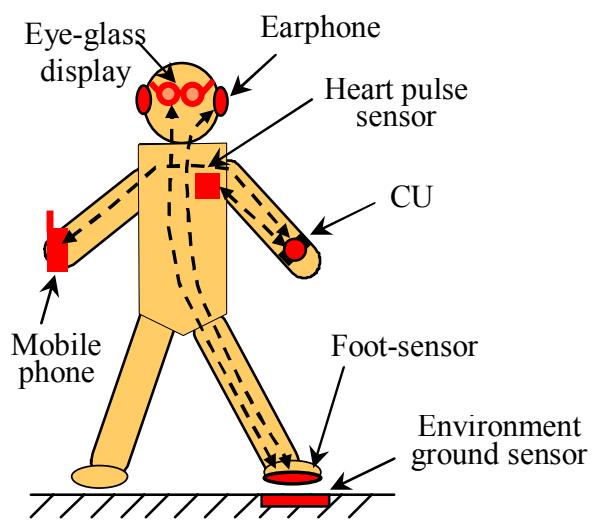

Figure 1. Example scenario of BAN devices distribution 
distance communications through e.g. WLANs and operators' infrastructures (cellular networks and satellite communications). As long as one device of the user's BAN has the capability for out-body communications, the rest of devices, even if they are only IBC enabled communicators, could communicate with the outside through the device with both IBC and out-body communication functions. In the following subsections, we explain the features of each element of the IBC system model.

\section{A. Central Unit $(C U)$}

We define CU as the master device of user's BAN. We think that user's wrist could be a good place to mount on the CU. The main features of this master device are:

- CU should serve as an intelligent hub for user's BAN, managing all the IBC enabled devices at anytime.

- CU should store information about user's personal data (i.e. preferences, interests, credit card information and identification passwords) and also properties of the IBC enabled devices.

- CU should detect when user touches or grasps an IBC enabled device as this action could mean that user wants to establish an IBC.

- CU should decide the most suitable active device of the user's BAN for the type of data to be sent.

- Because all the devices share the same body transmission medium and in order to allow only the selected device to receive the data, the CU should assign an identification code (IC) to each IBC enabled device when accessing to the user's BAN. At the same time, the wearable devices should be informed about the IC of the CU. Whenever a device wants to transmit information through the body, the CU should send the IC of the selected receiver (RX) to the TX. In this way, the TX could send the IC together with the data and, therefore, only the device that is identified by the IC could receive the information properly.

- CU should carry out not only IBC but also outside body communications, so that at least one device of the user's BAN could communicate with the outside.

\section{B. Wearable devices}

This group is composed of devices worn by the user such as mobile phone, PDA, notebook, eye-glass display, MP3 player, wireless pen and health monitoring sensors. Each gadget is designed for a different purpose with different features (e.g. eye-glass display is suitable for reading emails and watching videos but not for writing emails). Some of these devices could carry out both IBC and outside body communications (e.g. mobile phone) and others only IBC (e.g. eye-glass display). Wearable devices would be able to carry out both body-body IBC (i.e. communication between transceivers that are located in the body) and body-environment IBC (i.e. communication between a transceiver located in the body and another in the surrounding environment).

\section{Environment sensors}

This group is composed of elements that are located in the environment (e.g. in places such the ground, a wall and the knob of a door). Environment sensors could transfer data to the $\mathrm{CU}$, another IBC enabled device attached to the body or a processing unit located in the environment. These sensors would carry out body-environment IBC.

\section{IBC APPLICATIONS FoR HANDICAPPED PEOPLE}

We believe that IBC could be used to help handicapped people in their everyday life. Guidance, location based information and e-payment are some of the applications that we have foreseen for people with disabilities.

\section{A. Guidance and location based information}

Blind people could use IBC to be guided by means of voice information when walking in the streets, shopping centers and train stations. In order to perform this application, Fig. 2 illustrates that the blind person should wear an IBC enabled sensor located at the bottom of the shoe and an IBC enabled earphone to hear the information by voice. Moreover, an IBC enabling ground line should be embedded to the surface of the sidewalk. The IBC enabled ground line could send information such as the current location, the destination and time departure of the closest track to his/her position in a train terminal or special offers at the section of the supermarket where the blind person is at each moment. Whenever the blind person's shoes step the IBC enabled ground line, a communication link would be established between both IBC enabled foot sensor and ground. CU should detect the connection link and then inform the IBC enabled foot sensor about the IC of the IBC enabled earphone, so that the IBC enabled foot sensor could forward the data received from the ground sensors plus the IC to the IBC enabled earphone.

Deaf people could also take advantage of IBC and e.g. receive the information that is transmitted through the speakers in the train coaches or in train stations by means of text information that could be shown in an IBC enabled eye-glass display that the deaf person should wear. The communication procedure would be similar to the case explained previously for the blind people.

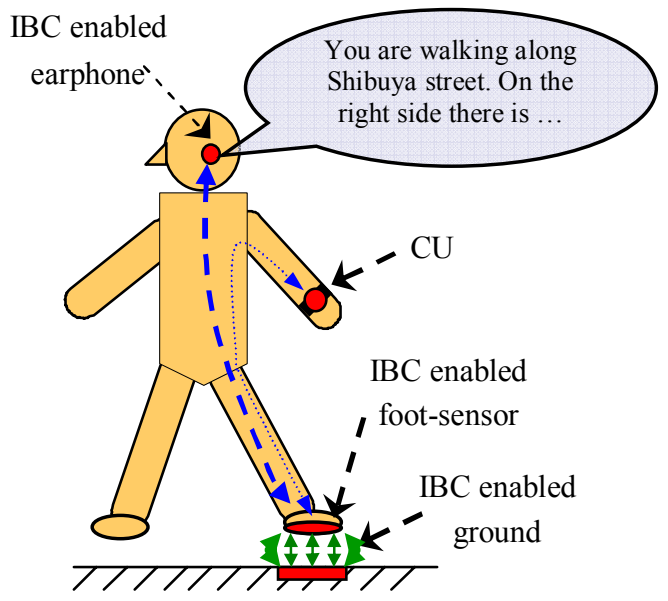

Figure 2. Guidance application scenario for blind people. 


\section{B. E-payment}

Electronic payment could be another application suitable for blind people so that e.g. they would not need to purchase a train ticket in the vending machines. To perform this application, sensors should be installed all along the ground of the ticket gate. The purpose of the ground sensors would be the detection and verification of credit card information while the blind person was walking through the gate. This process should be fast enough, so the blind person would not need to stop and wait until the verification of the credit card is finished to go through the gate.

Regarding the communication procedure, when the blind person was walking through the ticket gate, firstly, the CU should detect that a sensor worn in his/her foot is stepping a ground sensor with e-payment detection function. Next, the credit card information plus the IC of the IBC enabled foot sensor would be sent from the CU to the foot sensor and, then, to the IBC enabled ground. Finally, the gate would open after verification of the credit card.

\section{IBC APPLICATIONS IN TRAIN COACHES}

In Japan, people usually spend a lot of time in trains to commute to the workplace, schools, etc. Moreover, passengers should disconnect their mobile phones in the reserved seat area of train coaches (i.e. people with disabilities, older people and pregnant women), and refrain from talking by phone in other areas of the coaches. Based on these premises, we propose two IBC applications to be employed in train coaches: personalized advertisement system and intra-body internet service. Note that these applications could be used not only in train coaches but also in other scenarios such as restaurants, department stores and conference rooms.

\section{A. Personalized Advertisement System}

We propose a new way of dynamically personalized advertising system by using IBC in trains (see Fig. 3). Passengers when touching a handle inside a train coach could download e.g. advertisements in the most suitable format (audio, text or video) for the type of wearable device available. In Tokyo, the train coaches have above the handle a small plastic covering the strap of the handle (what we call advertising device, AD), where static ads are inserted. If this device would have embedded an IBC enabled small system-ona-chip where the company (e.g. travel agency) could store several type of data (such as text, audio and video) for several type of devices with commercial information addressed to different kind of potential customers (i.e. students, businessmen, retired people, ...), then, when the passenger touches the handle with his IBC enabled wristwatch (i.e. the CU of passenger's BAN), communication could be established between the $\mathrm{CU}$ and the $\mathrm{AD}$. At the same time, the CU, which stores some information about passenger's interests and preferences, could help the AD to decide what kind of contents could be more suitable for the passenger (if student, e.g. cheap airplane ticket offers), in case that he/she really wanted to receive any information. Depending on the type of wearable devices available, the $\mathrm{CU}$ would ask the $\mathrm{AD}$ for the most suitable type of data for the most suitable device and then it

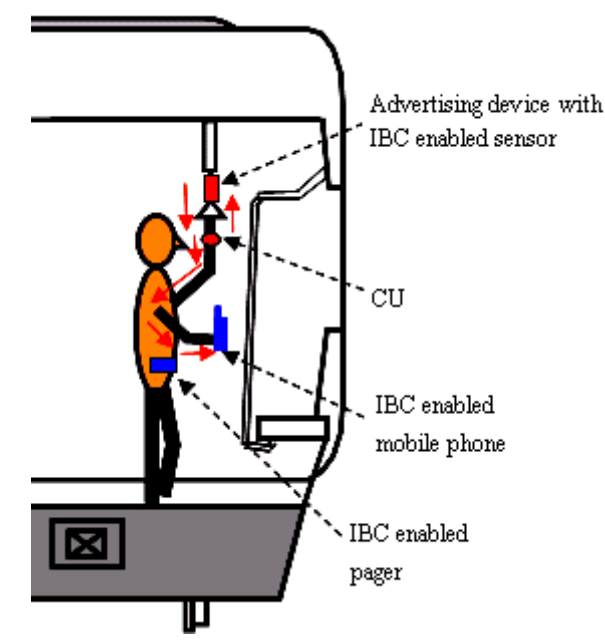

Figure 3. Cross section of half train coach showing the personalized advertisement application.

would resend the information to the selected device. For example, in Fig. 3, a mobile phone, a pager and the CU are the available devices. If the AD had stored text and video files, then the CU would select video data as the most suitable information for the mobile phone (because of the biggest display size among the three devices). Because all the wearable devices would share the same transmission medium, the CU would resend the data from the AD plus the IC of the selected device, so only the device identified by this code would be able to receive the information correctly.

\section{B. Intra-body Internet Service}

We define intra-body internet as a service whereby passengers could navigate internet, send/receive emails and even talk by means of their wearable devices (such as mobile phones, PDA and notebooks) not by using cellular networks but IBC. In order to perform this service, Fig. 4 shows that train coaches should need to install IBC enabled sensors in the ground. These sensors would be connected to a RF base station

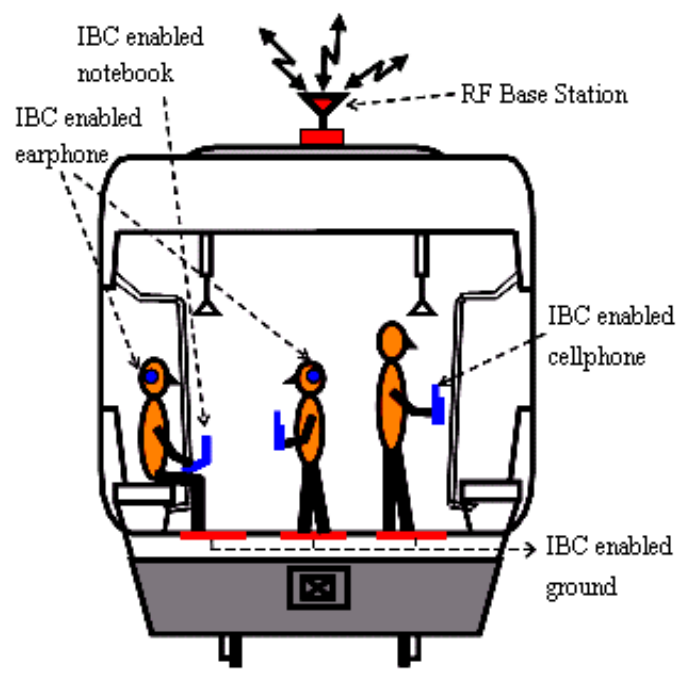

Figure 4. Cross section of train coach showing the intra-body internet service scenario. 
(BS) located on the top outside the coach. Whenever passengers wanted to navigate the internet, send/receive emails or talk with a friend, their IBC enabled devices would establish a communication link with the IBC enabled ground through their IBC enabled foot sensor. Next, the data would be transferred to the BS that would be in charge of providing the communication with the outside. The use of passengers' BAN devices would be allowed inside the trains as these devices would not utilize high radio frequency signals to communicate with cellular networks.

It is possible that several passengers would want to send or receive data at the same time, sharing the same IBC enabled ground. Therefore, in order to transfer the data of each passenger from/to the BS, people would need to be identified by a passenger identification code (PIC) that would be set up between each passenger's CU and the BS. Moreover, CU would inform to the BS about basic passenger's personal access information such as telephone number, email address and IP number, so whenever some data comes from the outside, the BS could identify the data of the passenger whom it should be transfer. At the same time, BS would inform to the passengers' $\mathrm{CU}$ about its identification code (BSIC). This process would be carried out when the passenger enters the coach, so the BS would be able to manage the active passengers with IBC enabled devices.

Both downlink and uplink communications could be performed. In case of uplink communication, when e.g. a passenger wants to send an email, firstly, the $\mathrm{CU}$ should inform the device willing to send the email (e.g. the IBC enabled notebook from Fig. 4), about the BSIC. Next, the data and the BSIC would be transmitted from the notebook to the IBC enabled ground sensors through the IBC enabled foot sensor, and then to the BS. Finally the BS would send the email to the outside. On the other hand, in case of downlink, firstly, the BS should identify whether the received data belongs to one of the passengers in the coach. If so, BS would send the data (e.g. an email with a MP3 file attached) and the PIC of the addressee (e.g. in Fig. 4, the passenger in the middle) to the IBC enabled ground sensor area where the passenger would be located. Next, the data would be transfer from the IBC enabled ground to the passenger's foot sensor and then, based on the determination of $\mathrm{CU}$, the email would be transfer to the most suitable IBC enabled device, i.e. the earphones, so that the passenger could hear the MP3 file.

\section{Characterization Of Intra-Body Propagation CHANNEL}

In [4], the frequency and time domain responses were measured to investigate the transmission characteristics of the human body. The time domain response was composed by a strongest echo and some lower power echoes that could represent the received power of the direct path component and the reflected signal components respectively. Therefore, we suppose that the Rice distribution could be employed to describe statistically the intra-body propagation channel.

In this section, we aim to characterize the intra-body channel in terms of the Ricean $K$-factor which is defined as the ratio of the average power of the direct path to the average power of the reflected components. Note that $K$-factor is a good indicator of the channel quality.

In order to extract the $K$-factor, first we measure the instantaneous received power over 4 different distances between TX (located in one hand) and RX (located in both arms) and considering the test person not moving and walking. The experiments were performed in a classroom. The input signal power was selected based on [5] in which it is recommended a basic limit exposure of $0.08 \mathrm{~W} / \mathrm{kg}$ for the human body. We employed an input power of just $12 \mathrm{dBm}$ that is lower than the $37 \mathrm{dBm}$ maximum signal power that could be transmitted in case of considering an average body weight of $65 \mathrm{~kg}$. TX and RX were configured by a $2 \mathrm{~cm}$ diameter signal electrode touching the skin and a $10 \mathrm{~cm}$ by $5 \mathrm{~cm}$ ground circuit board distant $1.4 \mathrm{~cm}$ from the signal electrode [4]. The $200 \mathrm{MHz}$ frequency carrier was selected based on the suitable frequency range for IBC determined in [4]. Note that the same input power, the same frequency carrier and the same transceiver configuration were used in Section VI.

Fig. 5 illustrates an example of the time variation signal at distances of $20 \mathrm{~cm}$ and $60 \mathrm{~cm}$ for both static and walking test conditions. We observe that the instantaneous received power varies more significant in the case of the test person moving rather than in the case of the test person static. This means that, in the case of walking, the contribution of the multipath components is higher and, therefore, the quality of the communication link should be lower. Moreover, we observe that the local mean received power in distance $60 \mathrm{~cm}$ is lower than in $20 \mathrm{~cm}$ because of the longer path distance.

The $K$-factor is extracted from the instantaneous received power over 200 samples by using the well-known method of moments [6]. Fig. 6 illustrates the obtained values of $K$-factor as a function of the distance between TX and RX when the test person is static and walking. Both curves yield high values of $K$-factor which describes the high contribution of the direct path component. As we expected from the results of Fig. 5, when the test person is walking, the $K$-factor is lower than in the case of the static test condition. In general, increasing the distance between TX and RX yields a fall of the $K$-factor in both static and walking conditions. However, we observe that

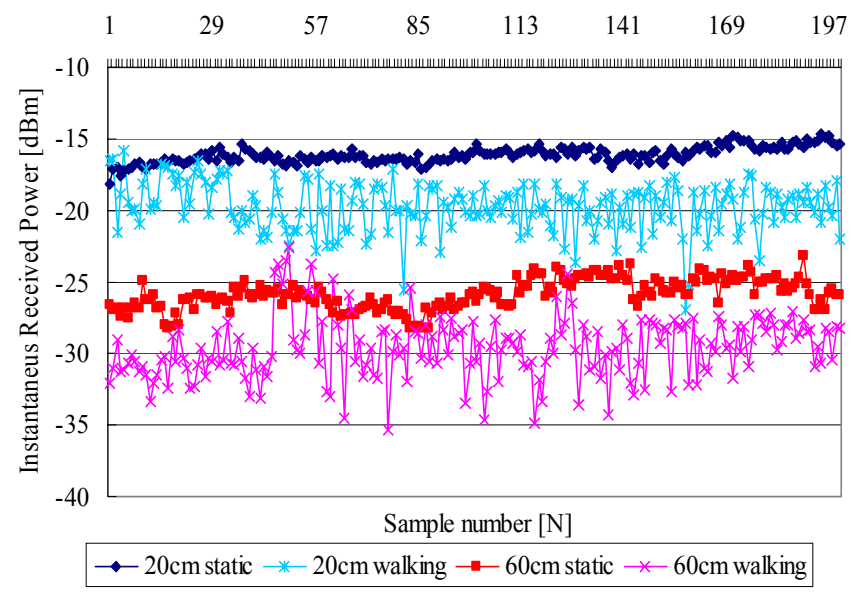

Figure 5. Received instantaneous power over 200 samples when distance between TX and RX is $20 \mathrm{~cm}$ and $60 \mathrm{~cm}$ and test person is static and moving. 


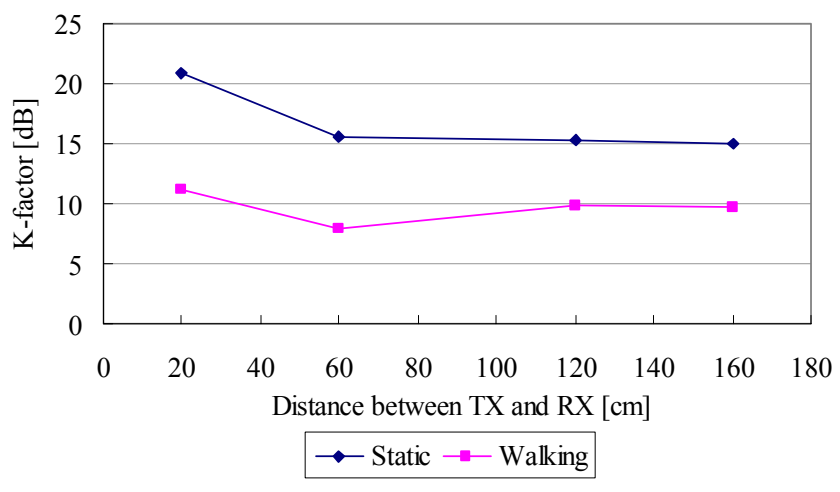

Figure 6. The measured $K$-factor when static and walking test conditions.

when walking there is a small rise for distances of $120 \mathrm{~cm}$ and $160 \mathrm{~cm}$. Both distances illustrate the scenario of the TX in the left hand and the RX in the right arm. When the transmitted signal from the left hand reaches the left shoulder, we believe that the direct path component still propagates to the right arm but some of the reflected components spread through the trunk and don't reach the right arm because of the low power level. If some of the reflected signals don't reach the RX, the ratio of the power of direct path to the power of the multipath components increases and, therefore, the $K$-factor increases.

\section{Evaluation Of Digital Modulation Schemes}

We investigate the performance of three digital modulation schemes that are commonly used in wireless systems. Constellation diagram and error vector magnitude (EVM) are measured to evaluate the performance of each modulation scheme considering several symbol rates and distances between TX and RX. The experiments were performed within the same environment as in Section V. A signal generator generated and modulated radio signals that were transmitted by a TX through the human body. The received signal were detected, demodulated and evaluated in a wireless communication analyzer. MSK, QPSK and 16QAM were the modulation schemes analyzed in the measurements. Symbol rates from $100 \mathrm{Ksps}$ to $5 \mathrm{Msps}$ were employed in the experiments in order to analyze the maximum data rates achievable through each modulation scheme. The measurements were carried out considering distances between TX and RX from $20 \mathrm{~cm}$ that corresponds to a distance between the wrist and the hand, to $180 \mathrm{~cm}$ that fits in with a TX in the wrist and a RX in the foot.

Fig. 7 first presents the constellation diagram results of both intra-body propagation channel (Figs. 7(b), 7(e) and 7(h), ) and air propagation channel (Figs. 7(a), 7(d) and 7(g)) in order to compare the performance of both transmission mediums within the same experimental conditions when symbol rate (SR) is $500 \mathrm{Ksps}$ and the distance between TX and RX (DTR) is $90 \mathrm{~cm}$. The results show that the intra-body propagation channel yields better performance, in all modulation schemes, than the air propagation channel which is more affected by distortion and external noises. Focusing on the results of the intra-body propagation channel, Fig. 7(b) shows that MSK provides the best performance as the received symbols are very close to the

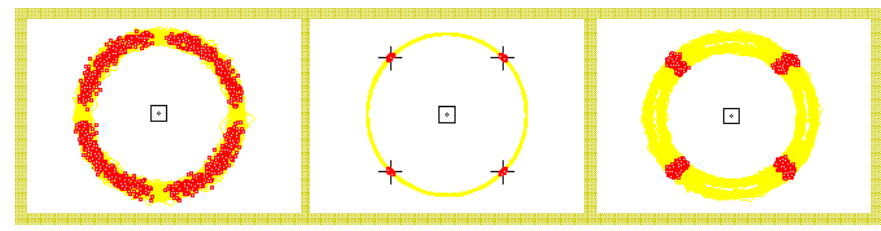

(a)

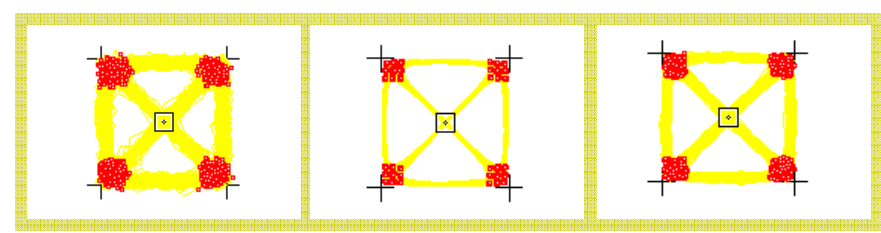

(d)

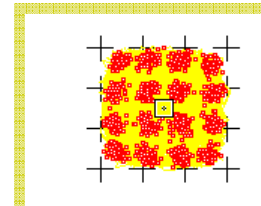

(g) (e)

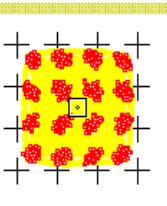

(i) (f)

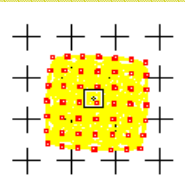

(h)
Figure 7. Constellation diagram results: (a) $\mathrm{MSK}$ air channel $\mathrm{SR}=500 \mathrm{Ksps}$ \& DTR $=90 \mathrm{~cm}$, (b) MSK intra-body channel SR=500Ksps \& DTR=90cm, (c) MSK intra-body channel $\mathrm{SR}=1 \mathrm{Msps} \& \mathrm{DTR}=180 \mathrm{~cm}$, (d) QPSK air channel $\mathrm{SR}=500 \mathrm{Ksps} \& \mathrm{DTR}=90 \mathrm{~cm}$, (e) QPSK intra-body channel $\mathrm{SR}=500 \mathrm{Ksps} \&$ DTR $=90 \mathrm{~cm}$, (f) QPSK intra-body channel $\mathrm{SR}=1 \mathrm{Msps} \& \mathrm{DTR}=180 \mathrm{~cm},(\mathrm{~g})$ 16QAM air channel $\mathrm{SR}=500 \mathrm{Ksps} \& \mathrm{DTR}=90 \mathrm{~cm}$, (h) $16 \mathrm{QAM}$ intra-body channel $\mathrm{SR}=500 \mathrm{~K}$ sps $\& \mathrm{DTR}=90 \mathrm{~cm}$, (i) $16 \mathrm{QAM}$ intra-body channel $\mathrm{SR}=1 \mathrm{Msps} \& \mathrm{DTR}=180 \mathrm{~cm}$.

reference constellation points. The worst results are provided by 16QAM (see Fig. 7(h)) with received points far from the constellation points. On the other hand, Figs. 7(c), 7(f) and 7(i) illustrate the results of increasing the distance between TX and RX from $90 \mathrm{~cm}$ to $180 \mathrm{~cm}$ and the symbol rate from $500 \mathrm{Ksps}$ to $1 \mathrm{Msps}$ in the intra-body transmission medium. The increment of transmission rate and distance causes that the distortion also increases in all modulation schemes.

EVM is an important metric for testing the modulation accuracy. EVM quantifies the difference between the ideal signal and the measured signal. A large EVM degrades transceiver performance by causing poor detection accuracy and, therefore, low modulation quality. Mobile communication standards define EVM requirements for the employed modulation techniques. For example, GSM and 3G WCDMA set up that EVM shall not be worse than 7\% for MSK [7], $17.5 \%$ for QPSK [8] and $12.5 \%$ for 16QAM [8]. In the measurements, we take these requirements as the thresholds for our evaluations. Figs. 8, 9 and 10 present the EVM results in terms of the root mean square percentage and as a function of the symbol rate. Fig. 8 shows that MSK yields good results with EVM measurements below the EVM requirement for symbol rates up to $5 \mathrm{Msps}$ when distance is $20 \mathrm{~cm}$, symbol rates up to $2.5 \mathrm{Msps}$ when distance is $90 \mathrm{~cm}$, symbol rates up to 1 Msps when distance is $155 \mathrm{~cm}$ and symbol rates up to 500Ksps when the largest distance is considered. Figs. 9and 10 show respectively that the EVM results of QPSK and 16QAM are worse than the EVM requirements for all the symbol rates and distances considered in the measurements. 


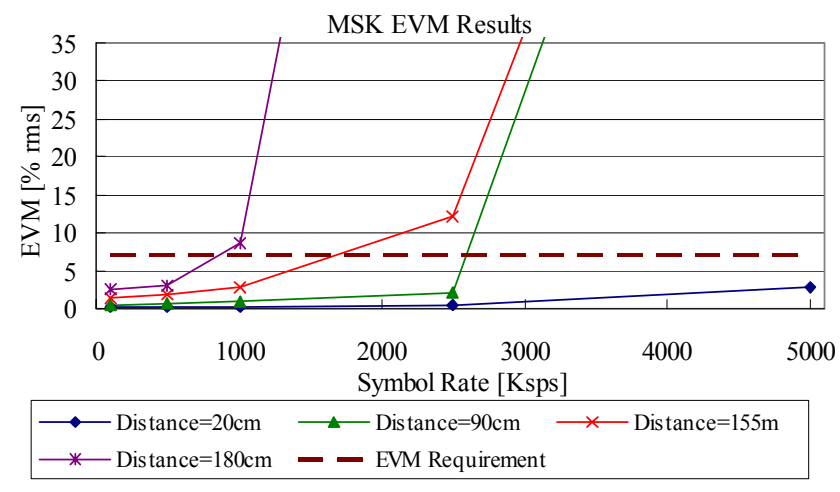

Figure 8. EVM results of MSK.

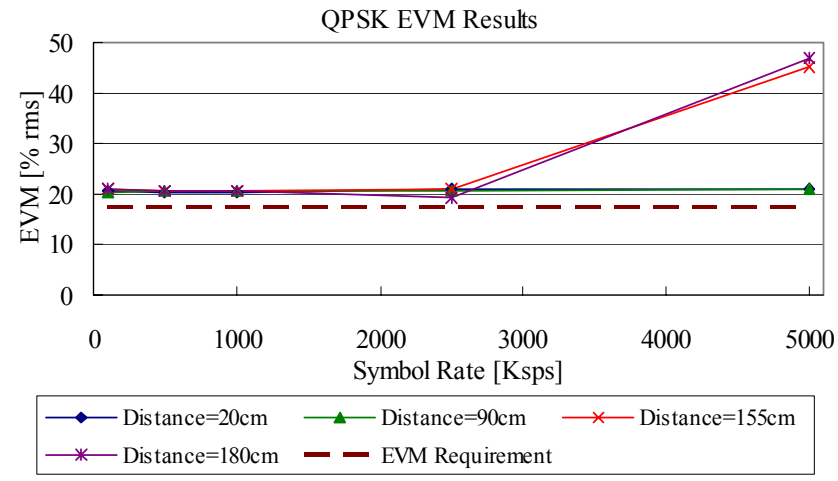

Figure 9. EVM results of QPSK.

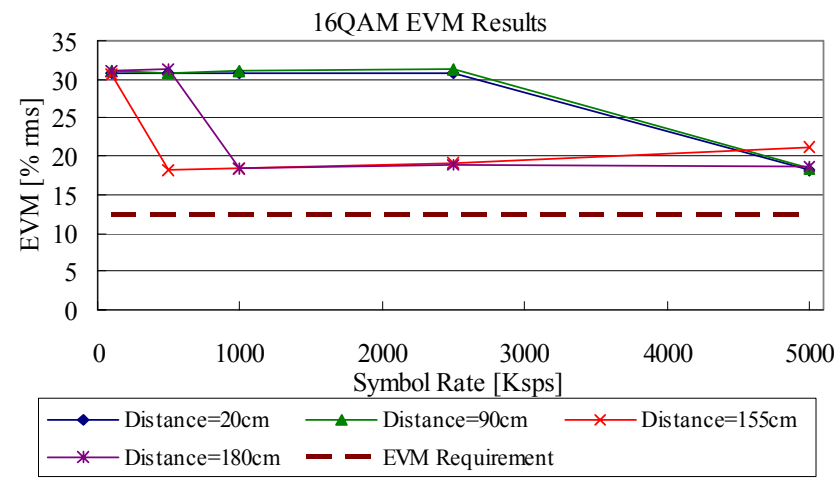

Figure 10. EVM results of 16QAM.

\section{CONCLUSIONS}

In the present paper, we have presented four novel applications employing IBC that we believe that it could be very useful for handicapped people and passengers in train coaches commuting to workplaces, schools, etc. Moreover, we have shown the system model in order to perform these IBC applications.

On the other hand, we have carried out experiments where firstly we have investigated the characteristics of the intra-body propagation channel and then we have evaluated three digital modulation schemes. The $K$-factor values extracted from the time varying instantaneous received power have shown that the quality of the IBC intra-body communication link is higher when the test person is not moving and the distance between TX and RX is shorter. The experimental evaluation of the modulation schemes has shown that MSK is the most suitable modulation scheme with the lowest EVM performance and the closest received symbols to the constellation points. Moreover, the results have proved that high transmission rates are achievable through IBC and, therefore, heavy data communications such as video streaming could be carried out through the human body.

\section{REFERENCES}

[1] T. G. Zimmerman, "Personal Area Networks: Near-filed Intra-Body Communication,” IBM Systems Journal, Vol .35, N. 3\&4, pp. 609-617, 1996.

[2] Matsushita Electric Works Ltd., 2004, www.mew.co.jp/epress/2004/0409-02.htm

[3] K. Fujii, K. Ito, "Evaluation of the Received Signal Level in Relation to the Size and Carrier Frequencies of the Wearable Device Using Human Body as a Transmission Channel," Proc. of 2004 Antennas and Propagation Society Symposium, pp. 105-108, June 2004.

[4] J. Agud Ruiz, X. Jiang, S. Shimamoto, "Propagation Characteristics of Intra-body Communications for Body Area Networks", IEEE Consumer Communications and Networking Conference 2006, Las Vegas, January 2006.

[5] World Health Organization, "Electromagnetic Fields $(300 \mathrm{~Hz}$ to 300GHz)," 1993, www.inchem.org/documents/ehc/ehc/ehc137.htm

[6] K. K. Talukdar and W. D. Lawing, "Estimation of the parameters of the Rice distribution", Journal of Acoustical Society of America, vol. 89, no. 3, pp. 1193-1197, 1991

[7] ETSI GSM 5.05 Standard v.8.4.1: "Digital Cellular Telecommunications System (phase 2+), Radio Transmission and Reception", 1999

[8] 3GPP TS 25.101 v.6.8.0: "User Equipment (UE) radio transmission and reception (FDD)", 